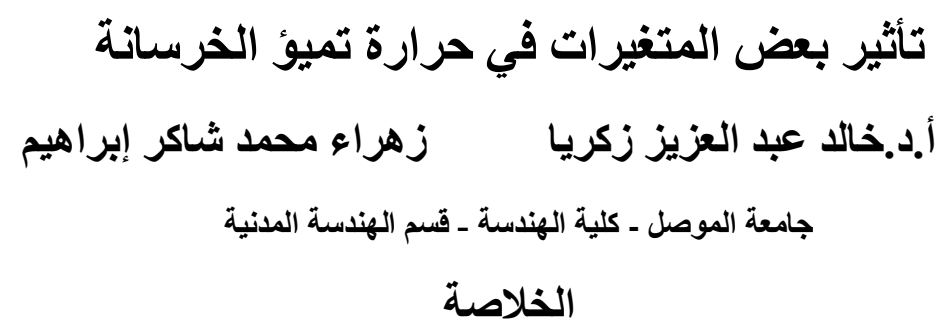

تم في هذا البحث دراسة تأثير بعض المتغيرات في حرارة التميؤ لنوعين من الخلطات الخرسانية، الأول خرسانة

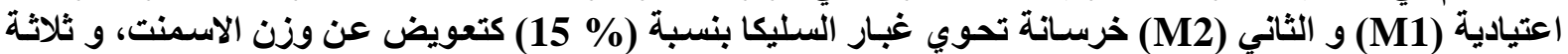

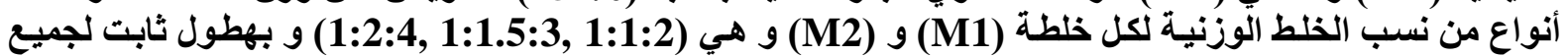

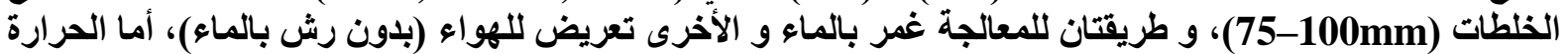

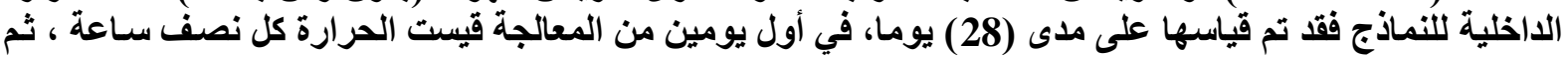

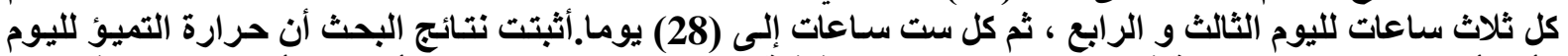

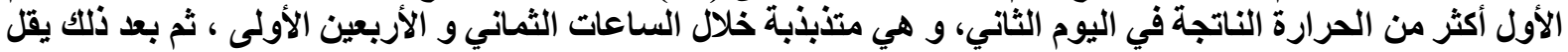

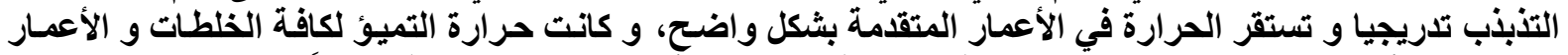

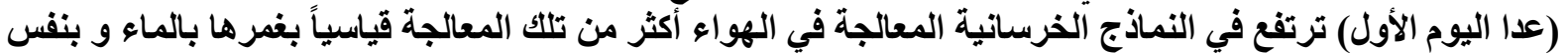

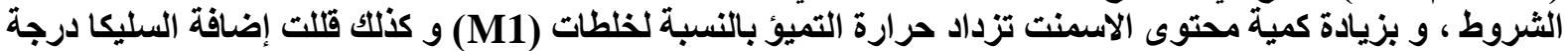

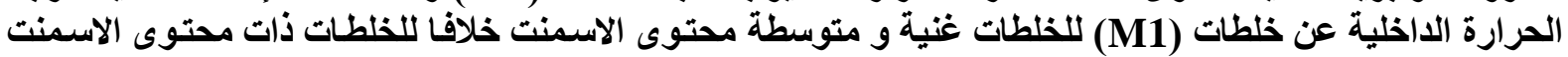

\title{
Effect of Some Variables in Heat of Hydration in concrete
}

\author{
Prof. Dr. Kh. A. Zakaria Z. M. Sh. Ibrahim \\ University of Mosul - College of Engineering, - Civil Eng. Dept.
}

\begin{abstract}
In this research, the effects of some parameters on the heat of hydration for two concrete mixes have been studied. The mixes are with and without $15 \%$ micro silica as a cement weight replacement designated (M2) and (M1) respectively, and Three concrete mixes by weight namely, $(1: 2: 4,1: 1.5: 3$, and 1:1:2) at uniform consistency that is a constant slump of $(75-100 \mathrm{~mm})$ for both(M1) and (M2), and Two curing methods, moist and air cured, While the internal temperature for the specimens was measured for (28) days : at the $1^{\text {st }}$ two days at intervals of half an hour then at intervals of ( $3 \mathrm{hrs})$ for the $3^{\text {rd }}$ and $4^{\text {th }}$ day, while at intervals of (6 hrs) until (28) days. The results of the research proved that the heat of hydration was higher at the $1^{\text {st }}$ day than that at the $2^{\text {nd }}$ day, fluctuating during the $1^{\text {st }}(48 \mathrm{hrs})$. The fluctuation reduces gradually to a steady rate at later ages, While the heat of hydration for all mixes and at all ages (except the $1^{\text {st }}$ day) was higher for air cured specimens than that moist cured for same condition, As the cement content increased the heat of hydration (for mixes M1) increased accordingly and also the addition of silica lowered the internal concrete temperature for M1 mixes (at high and moderate cement contents) in opposite of few cement contents.
\end{abstract}

$$
\text { قبل: } 2012 \text { - } 5 \text { - } 6
$$

22 أستلم: 2012 - 22 
يتطور تميؤ الاسمنت إذا توفرت معالجة مناسبة بحيث بيئة المعالجة رطبة و دافئة و بهذا تقل المسامية و تزداد المباد

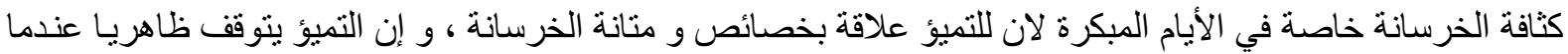

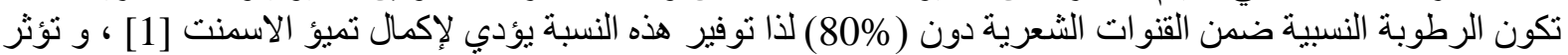
في حرارة التميؤ العديد من العو امل منها :

$$
\begin{aligned}
& \text { 1. نسبة (الماء/الاسمنت). } \\
& \text { 2. . نسبة (الركام /الاسمنت). } \\
& \text { 3. نوع الاسمنت. } \\
& \text { 4. حجم النموذج وشكله. } \\
& \text { 5. . ظروف المعالجة. } \\
& \text { 6. عر الخرسانة عند الفحص. }
\end{aligned}
$$

يفسر تميؤ الاسمنت الذي يحدث داخل الخرسـانة على أنه تفاعل كيميائي مصحوب بحرارة (Exothermic) موضحاً في الخطوات الآتية:

$$
\begin{array}{r}
-1 \\
-2 \\
-3
\end{array}
$$

Ca plus H-O-H (Water) $\rightarrow$ CaoH plus H + (released energy)

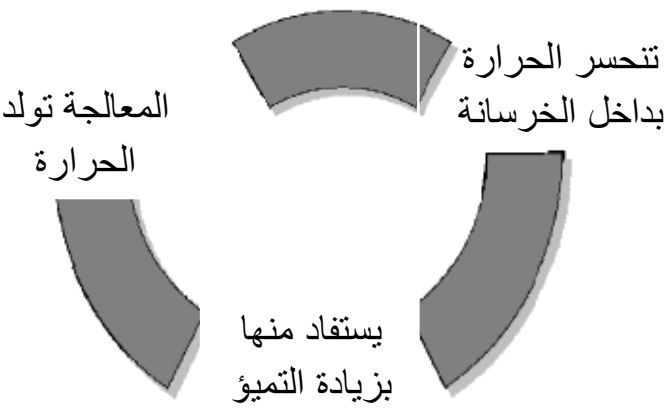

الثكل (1) : دورة معالجة الخرسانة [2].

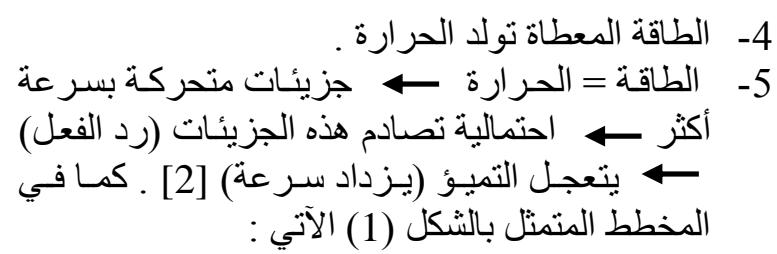

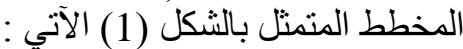

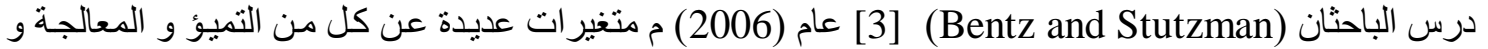
بنية (Microstructure) عجينة الاسمنت باستخدام عدة طر ائق ؛ منها طريقة (Scanning electron microscopy)

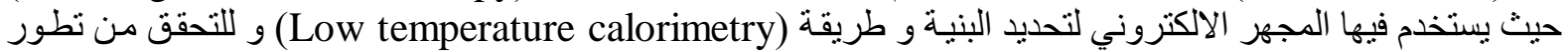

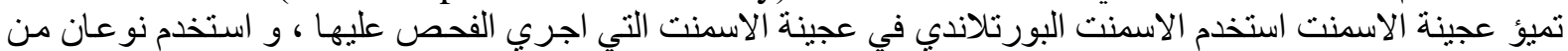

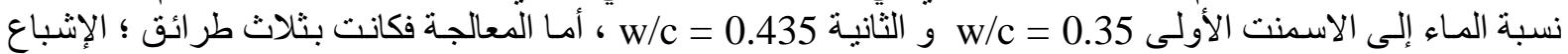

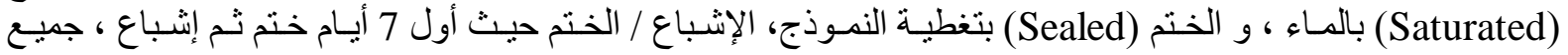
المعالجات كانت بدرجة حرارة 20 مغرفة معزولة، و قد ظهر أن المعالجة لها تأثير في كل من التميؤ و البنية للمنشأ، و

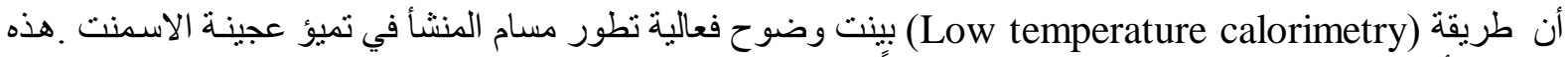

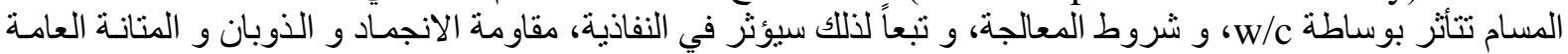




$$
\text { البرنامج العملي : }
$$

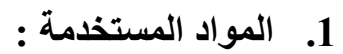

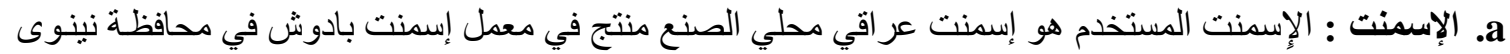

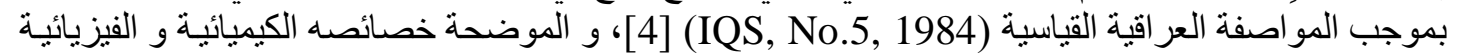
في الجدولين (1) و (2) على التوالي.

الجدول (1) : خصائص الاسمنت المستخذم.

الخصائص الكيميائية

\begin{tabular}{|c|c|c|c|c|c|c|}
\hline $\mathrm{Al}_{2} \mathbf{O}_{3}$ & $\mathrm{SiO}_{2}$ & $\mathrm{Fe}_{2} \mathrm{O}_{3}$ & $\mathrm{CaO}$ & $\mathrm{SO}_{3}$ & MgO & اكاسيد عناصر الاسمنت \\
\hline 3.43 & 23.20 & 4.88 & 62.72 & 2.26 & 3.20 & النسبة المئوية (\%) \\
\hline & & & & $\begin{array}{l}\text { لا يزيد عن } 2.3 \% \\
\text { لا }\end{array}$ & 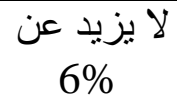 & حدود المواصفة \\
\hline \multicolumn{2}{|c|}{$\mathrm{C}_{3} \mathrm{~S}$} & $\mathrm{C}_{2} \mathrm{~S}$ & $\mathrm{C}_{3} \mathrm{~A}$ & \multicolumn{2}{|c|}{$\mathbf{C}_{\mathbf{4}} \mathbf{A F}$} & المركبات الرئيسية \\
\hline \multirow{2}{*}{\multicolumn{2}{|c|}{42.58}} & 34.39 & 0.82 & \multicolumn{2}{|c|}{14.85} & النسبة المئوية (\%) \\
\hline & & & عن لا يزيد & & & حدود المواصفة \\
\hline
\end{tabular}

الجدول (2) : خصائص الاسمنت المستخدم .

الخصائص الفيزيائية

\begin{tabular}{|c|c|c|c|c|c|}
\hline \multicolumn{2}{|c|}{ مقاومة الانضغاط (MPa) } & \multicolumn{2}{|c|}{ وقت التماسك } & \multirow[t]{2}{*}{ 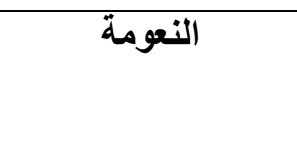 } & \multirow{2}{*}{ الخصائص } \\
\hline 7 & 3 & النهائي (دقيقة) & الابتدائي (دقيقة) & & \\
\hline 26.8 & 18.4 & 300 & 120 & $4 \%$ & نتيجة الفحص \\
\hline $\begin{array}{c}\text { لا تقل عن } \\
\text { (24 MPa) }\end{array}$ & $\begin{array}{c}\text { لا تقل عن (16 MPa ) } \\
\text { (16 }\end{array}$ & لا تززيد عنة & لا تقل عن 45 & 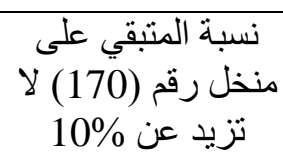 & حدود المواصفة \\
\hline
\end{tabular}

الماء : استخدم ماء الثرب الاعتبادي لمدينة الموصل في الخلطة الخرسانية، إذ إن جميع المواصفات تشبر إلى أن ماء

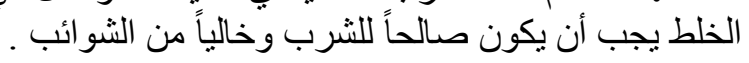

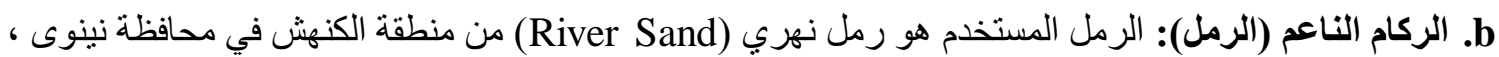

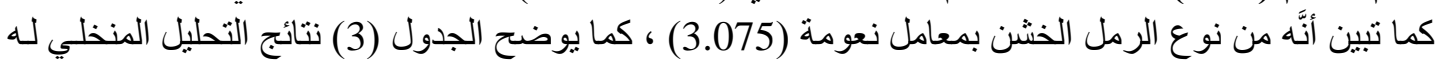

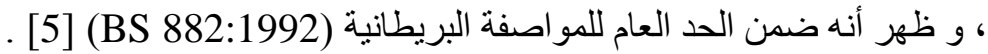

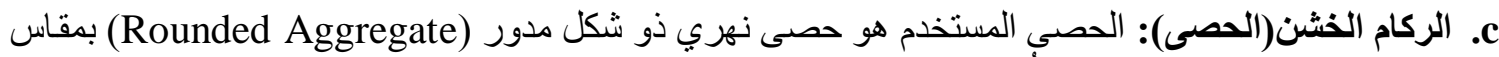

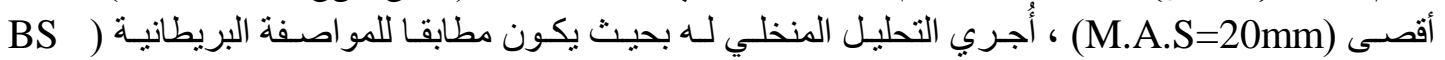
(582:1992) [5 . و الجدول (4) يوضح نتائج النحليل المنظلي لهي 
الجدول(3) : نتائج التحليل المنظلي للرمل المستخدم مع النسب المارة القياسية للمواصفة

(البر بطانبة (B.S 882:1992)

\begin{tabular}{|c|c|c|c|c|c|}
\hline \multicolumn{5}{|c|}{ النسبة المئوية المارة } & \multirow{3}{*}{ مقاس المنخل } \\
\hline \multicolumn{3}{|c|}{ حدود مناطق التدرج } & \multirow{2}{*}{ اللمواصفة الكلية } & \multirow{2}{*}{ للرمل المستخدام المارة } & \\
\hline ناعم F & متوسط M & خشن C & & & \\
\hline- & - & - & $100-89$ & 100 & 5.00 \\
\hline $100-80$ & $100-65$ & $100-60$ & $100-60$ & 73 & 2.36 \\
\hline $100-70$ & $100-45$ & $90-30$ & $100-30$ & 58 & 1.18 \\
\hline $100-55$ & $80-25$ & $54-15$ & $100-15$ & 43 & 0.60 \\
\hline $70-5$ & $48-5$ & $40-5$ & $70-5$ & 15 & 0.30 \\
\hline - & - & - & $15-0$ & 3.5 & 0.15 \\
\hline
\end{tabular}

الجدول(4) : التحليل المنظلي للحصى المستخدم مع النسب المارة القياسية للمواصفة البريطانية

. (BS 882:1992)

\begin{tabular}{|c|c|c|}
\hline مقاس المنذل (الحدود القياسية المارة \% & (النسبة المارة للحصى المستخدم) \\
\hline 95 & $100-90$ & 14 \\
\hline 60 & $80-40$ & 10 \\
\hline 45 & $60-30$ & 5 \\
\hline 5 & $10-0$ & 20 \\
\hline
\end{tabular}

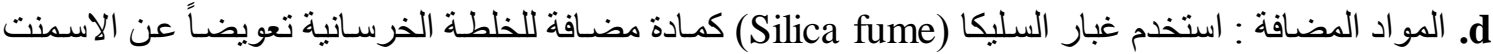

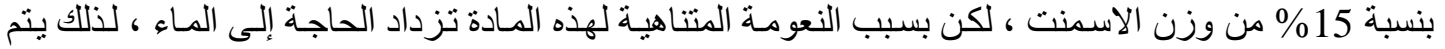

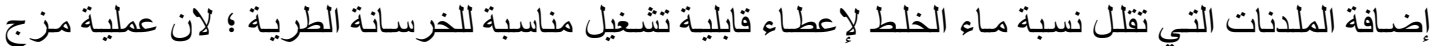

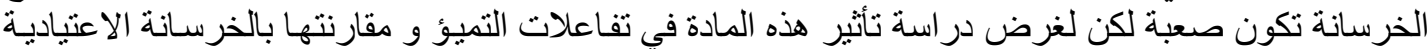

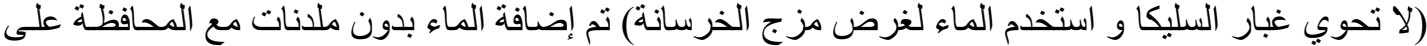

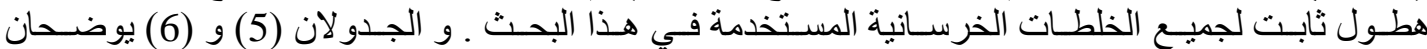

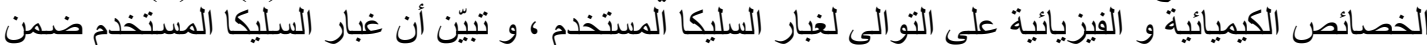

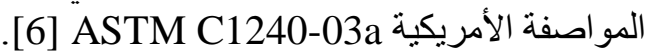

$$
\text { الجدول (5) : خصائص غب الخصائص الفيزيائية }
$$

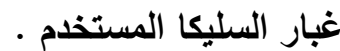

\begin{tabular}{|c|c|c|c|c|c|c|c|}
\hline $\mathrm{Na}_{2} \mathrm{O}$ & $\mathbf{A l}_{2} \mathbf{O}_{3}$ & $\mathrm{SiO}_{2}$ & $\mathrm{Fe}_{2} \mathrm{O}_{3}$ & $\mathrm{CaO}$ & $\mathbf{S O}_{3}$ & MgO & اكاسيد عناصر غبار السليكا \\
\hline 0.00 & 0.02 & 95.95 & 1.10 & 1.21 & 0.22 & 0.10 & النسبة المئوية (\%) \\
\hline & & لا ينقص عن 85 & & & لا يزيد عن 4 & & حدود المواصفة \\
\hline
\end{tabular}

الجدول (6) : خصائص غبار السليكا المستخدم .

الخصائص الكيميائية

\begin{tabular}{|c|c|c|}
\hline 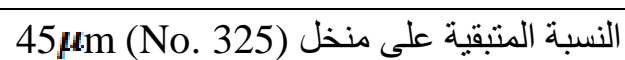 & المساحة السطحية (m²/Kg) & الخصائص \\
\hline 7 & 20000 & نتيجة الفحص \\
\hline $\max . \% 10$ & $\min .15000$ & حدود المواصفة \\
\hline
\end{tabular}




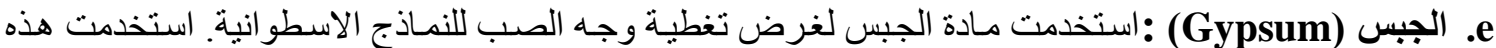

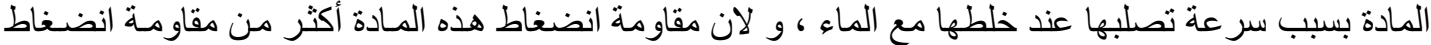

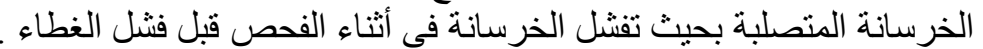

$$
\text { 2. }
$$

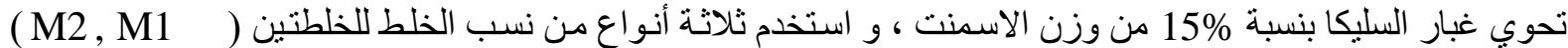

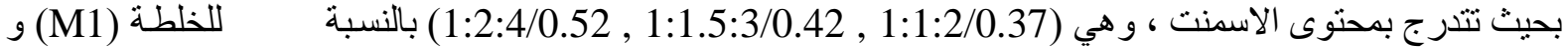

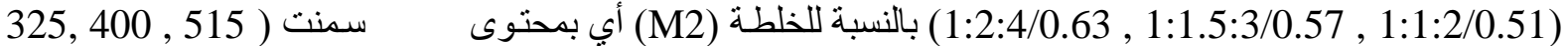

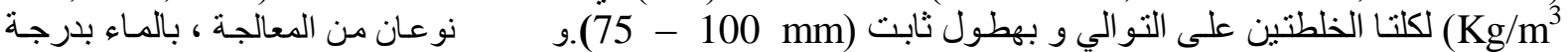

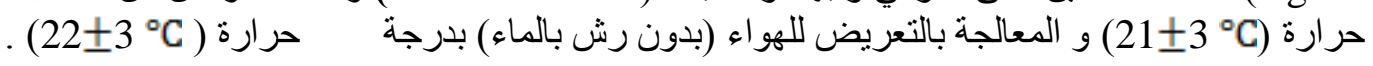

\section{3. النماذج و طريقة الفحص :}

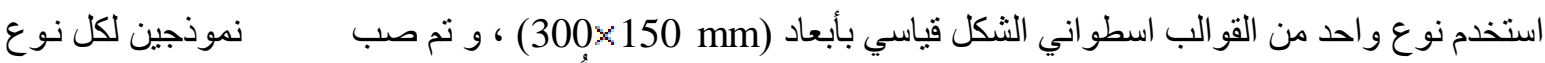

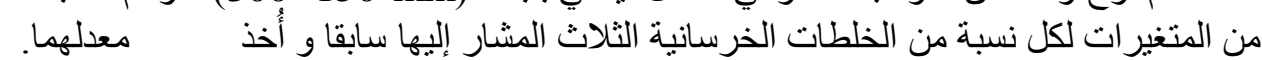

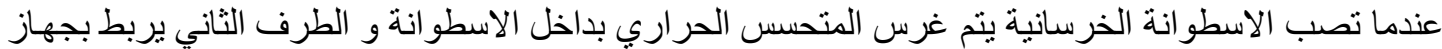

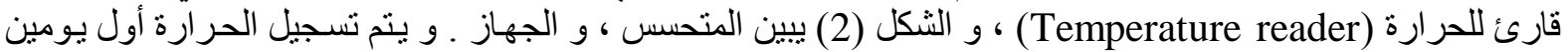

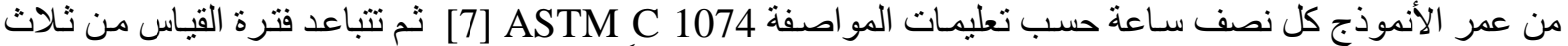

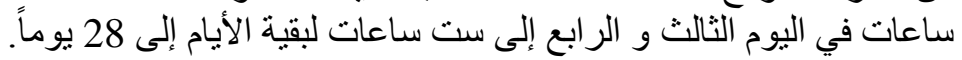

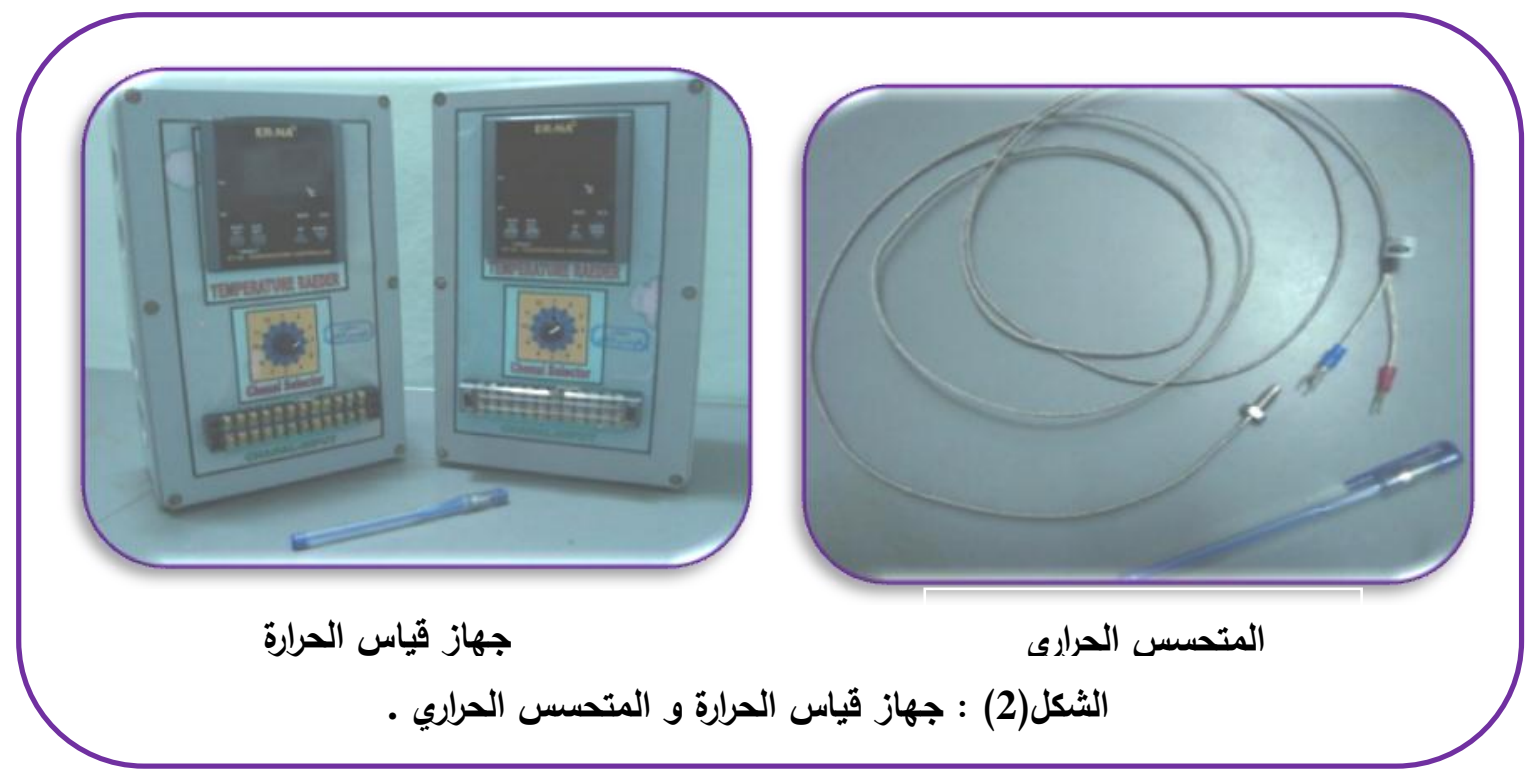

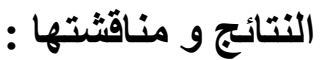

1 : تأثير عمر النماذج في حرارة التميؤ للنماذج الخرسانية :

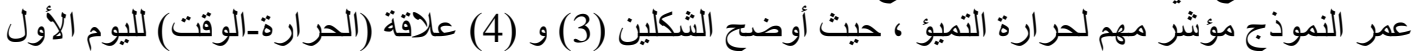

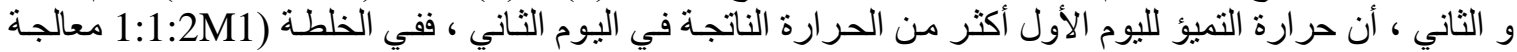
ماء) كان معدل درجة الحرارة في اليوم الأول (28.2

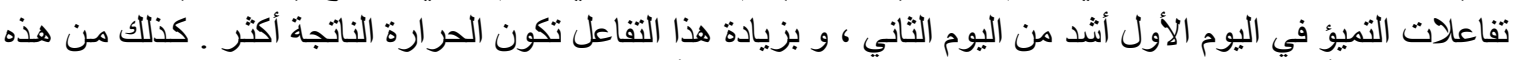

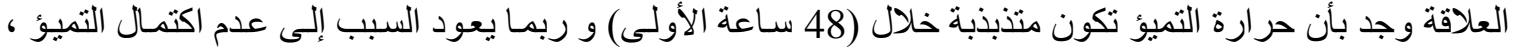

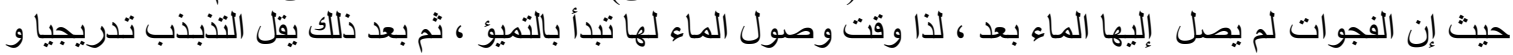


No. 2

April 2013

تستقر القراءة في الأعمار المتقدمة بشكل واضح ، ففي الخلطة المذكورة آنفاً تنتقر قراءة الحرارة الداخليـة تقريبا بين درجة حرارة (23 C 23 - 21.1) ؛ لان تفاعلات التميؤ تنتهي ، حيث يظهر هذا واضحا من خلال ملاحظة علاقات (الحر ارة_الوقت) القادمة (ارة)

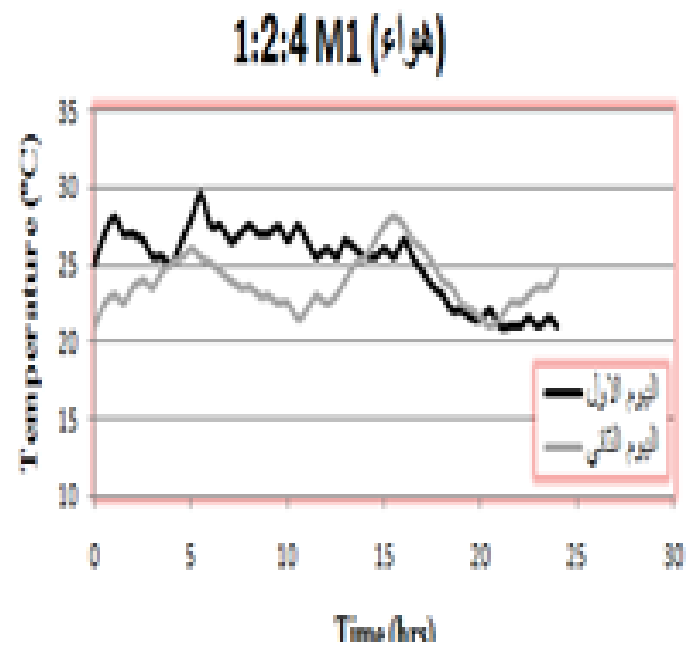

1:1.5:3 M1(4)

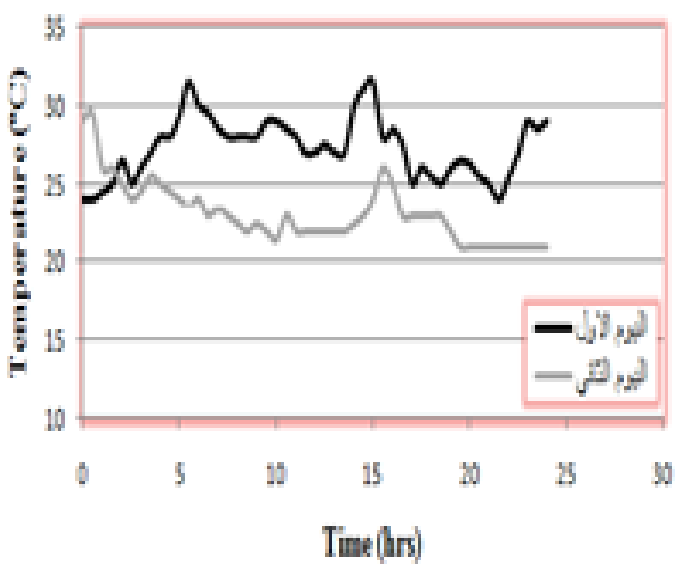

1:1:2M1(s|a)

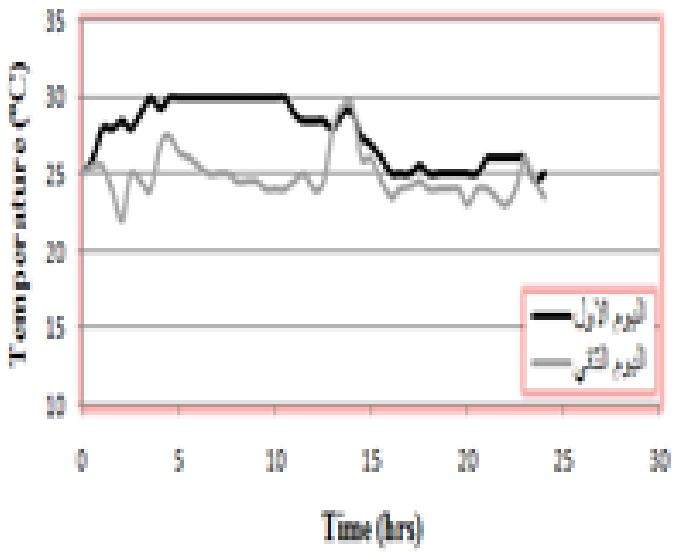

$1: 2: 4 \mathrm{M} 1(5 \mathrm{~s})$

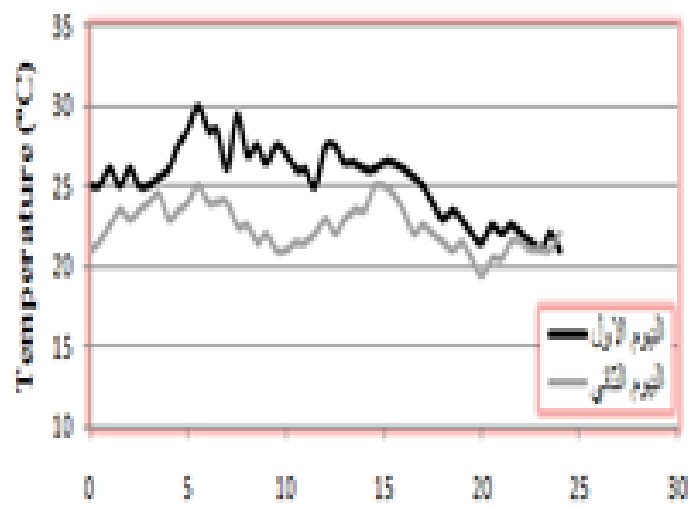

1:1.5:3 M1 $|q| 9 \mid$

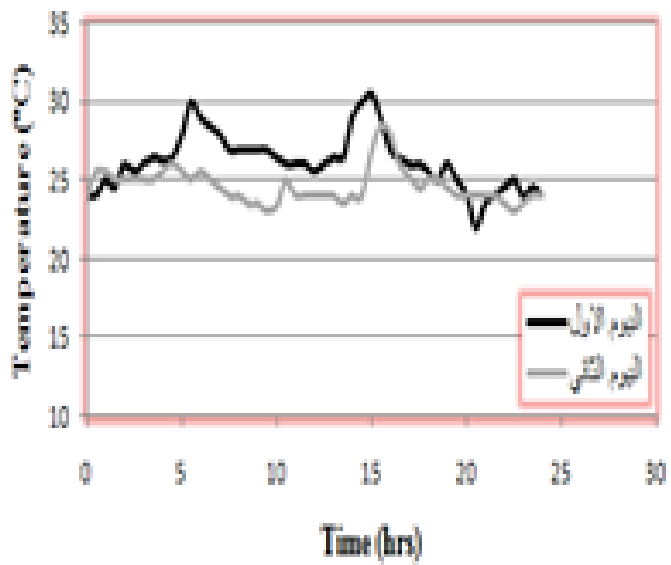

1:1:2M1(2)

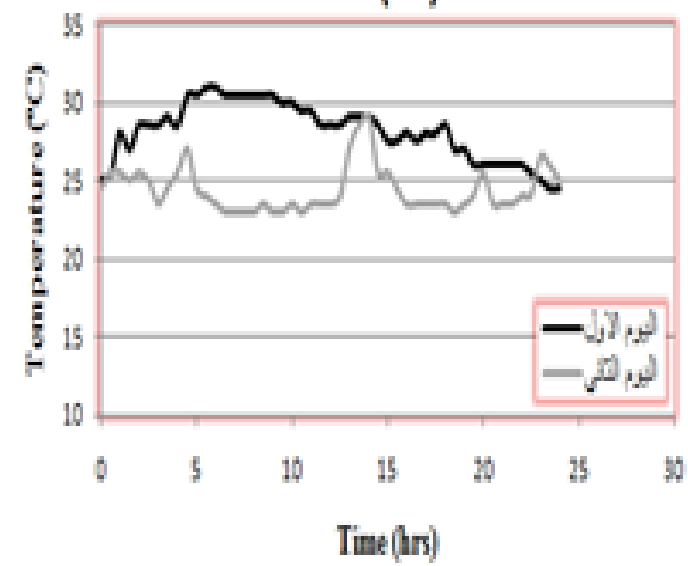

الثكل (3): علاقة (الحرارة- الوقت) لكل نوع من الخلطات الاعتيادية المدروسة. 
1:2:4 M2 (s) $(\mu)$

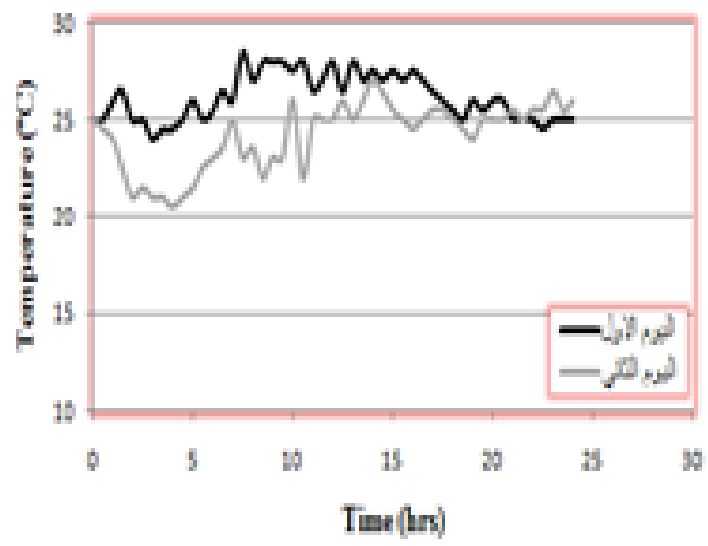

1:1.5:3 M2 (4) $(4)$

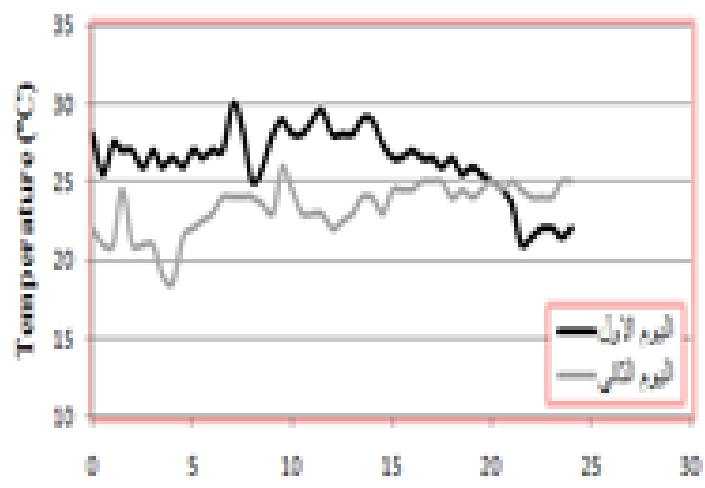

$\operatorname{Tim}(\mathbf{m})$

1:1:2M2 $2(\%|\alpha|$

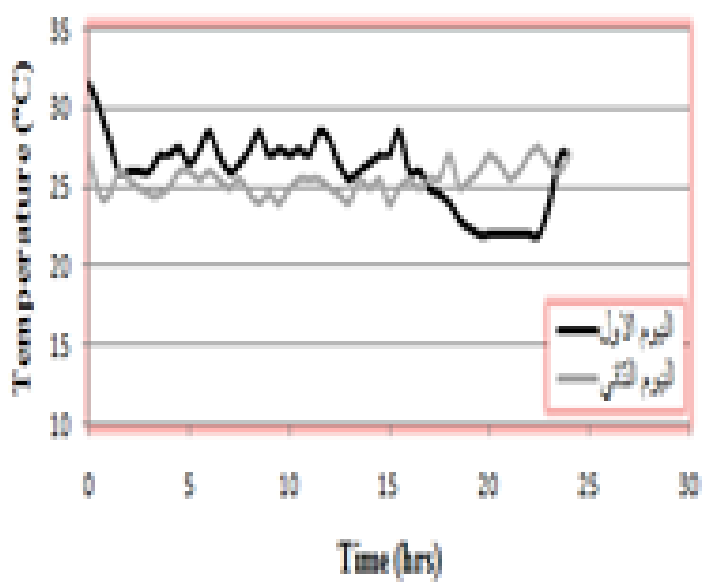

1:2:4M2 (4ئ)

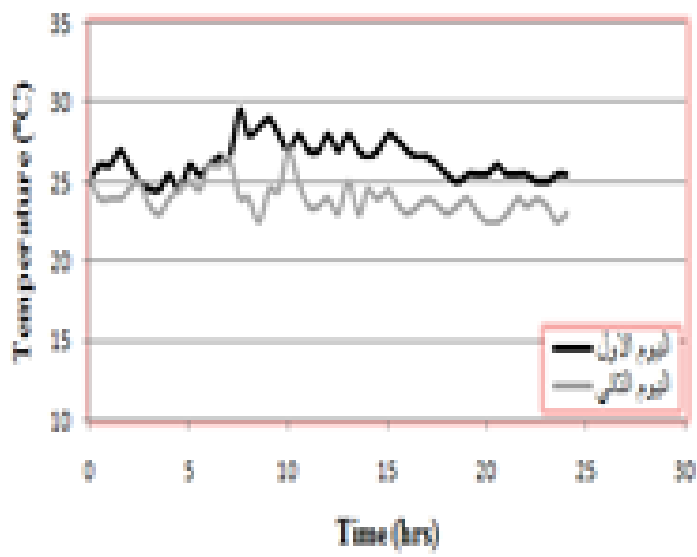

$1: 1.5: 3 \mathrm{M} 2(2 \mathrm{a})$

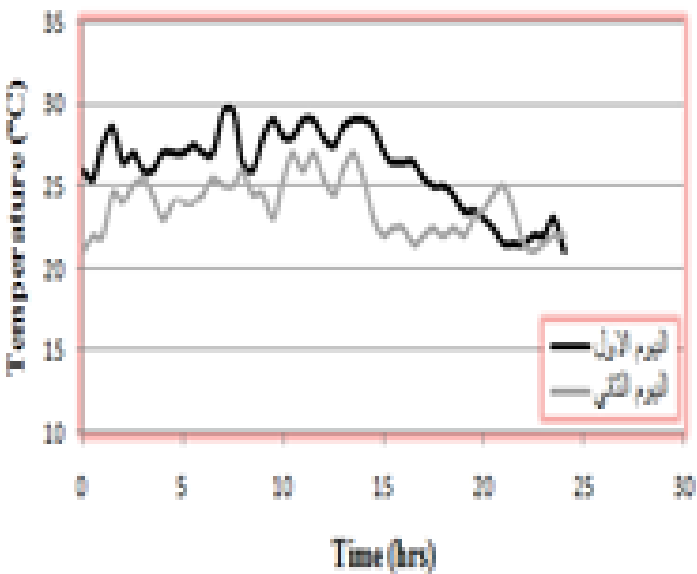

1:1:22 M2 2 (4)

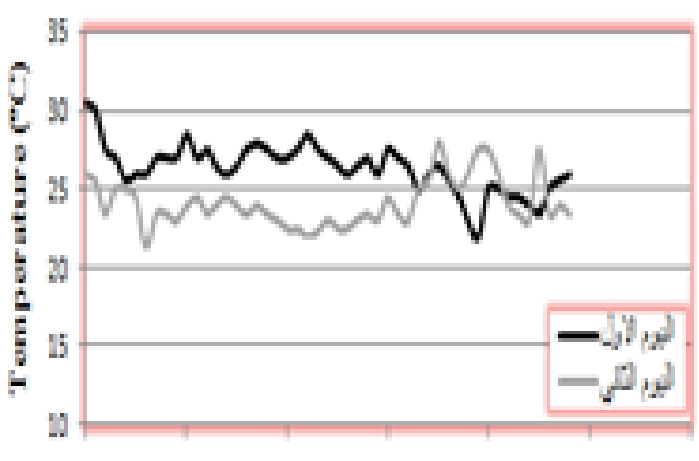

Time(bri)

الثكل (4): علاقة (الحرارة- الوقت) لكل نوع من الخلطات المدروسة الحاوية غبار السليكا بنسبة (\%) من وزن من الاسمنت 
2 : تأثير طريقة معالجة النماذج في حرارة التميؤ للنماذج الخرسانية :

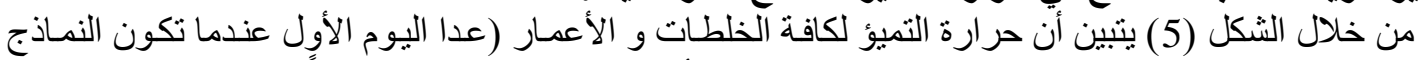

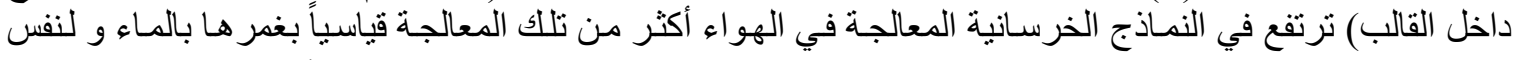

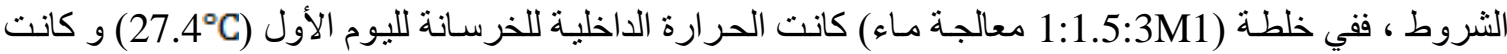

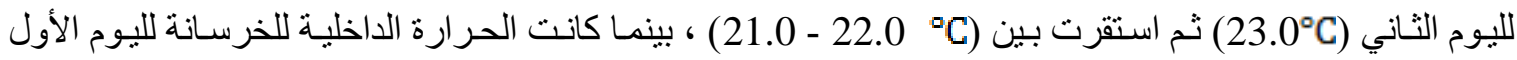

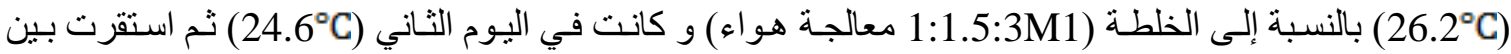

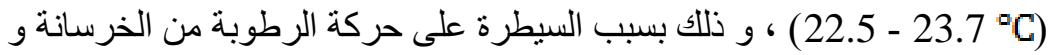

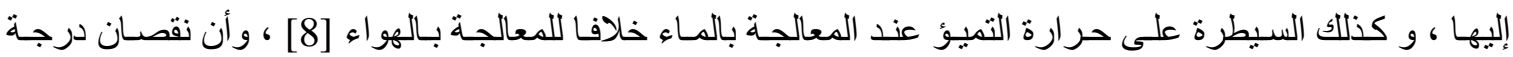
الحرارة عند المعالجة بالماء يبدو أكثر وضوة الإكا في الخلطات قليلة و معتدلة الاسمنت لان حر ارة تميؤه هـا اقل بالنسبة

3 : تأثير تغير محتوى الاسمنت في حرارة التميؤ للنماذج الخرسانية :

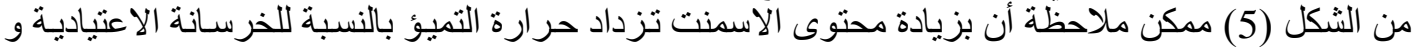

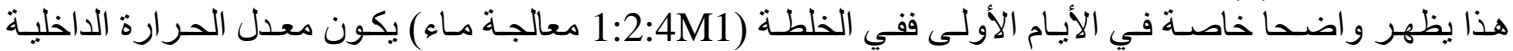

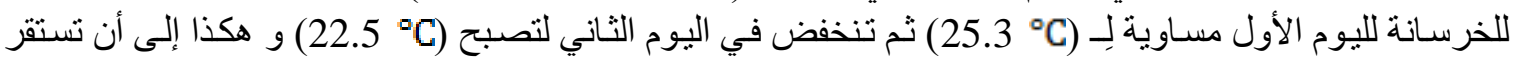

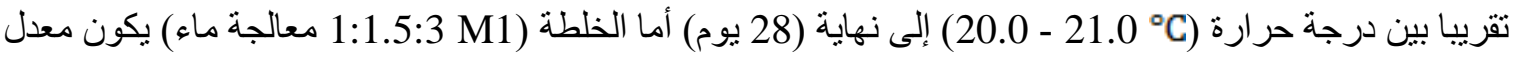

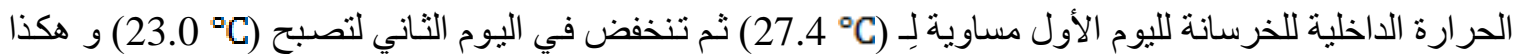

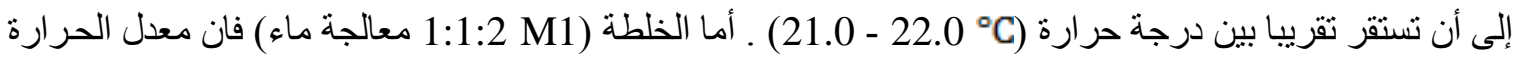

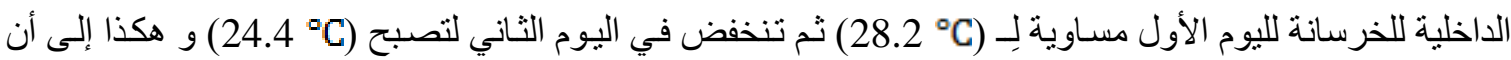
تستقر تقريبا بين درجة حرارة (23.0 23 - 21.1) ؛ و ذلك لأن زيادة محتوى الاسمنت تؤدي إلى زيادة تفاعلات

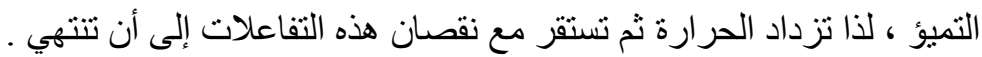

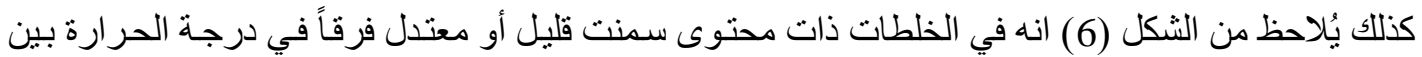

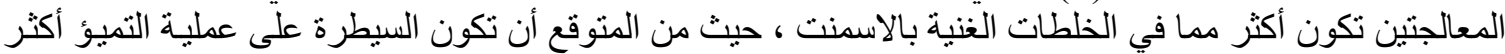

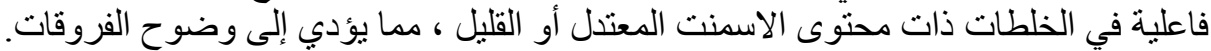

4 : تأثير وجود السليكا في حرارة التميؤ للنماذج الخرسانية :

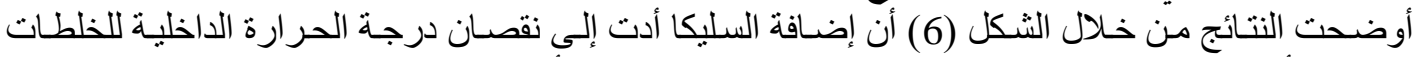

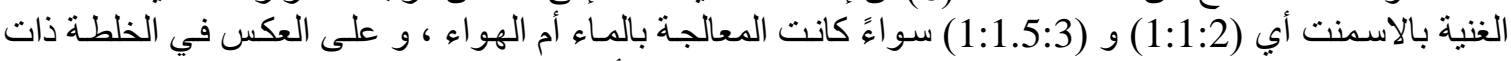

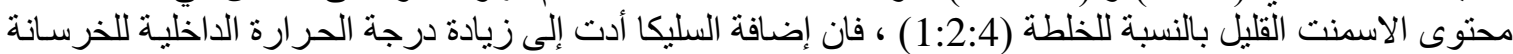

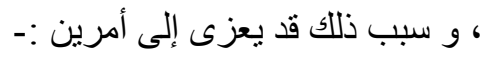
1.

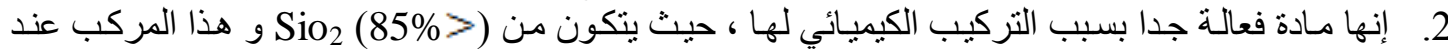

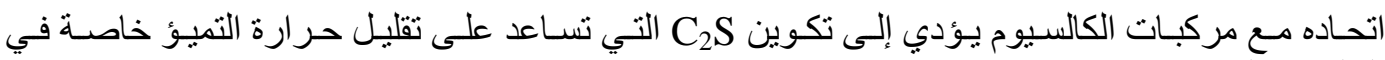

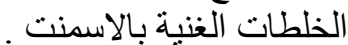


زكريا: تأثير بعض المتغيرات في حرارة تميؤ الخرسانة
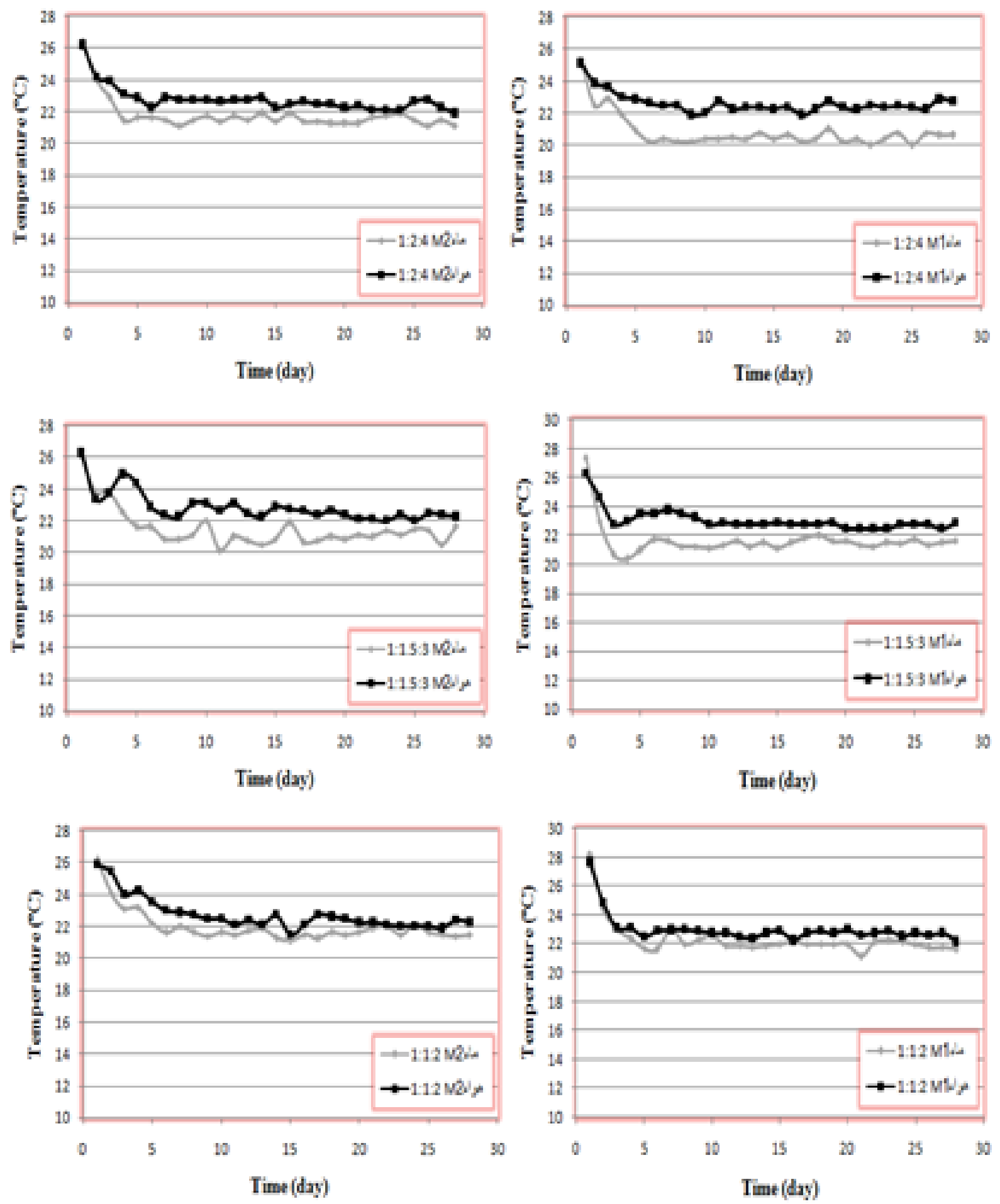

الثكل (5): تأثير طريقة المعالجة في حرارة تميؤ الخرسانة لكل نوع من الخلطات المدروسة . 
No. 2

April 2013
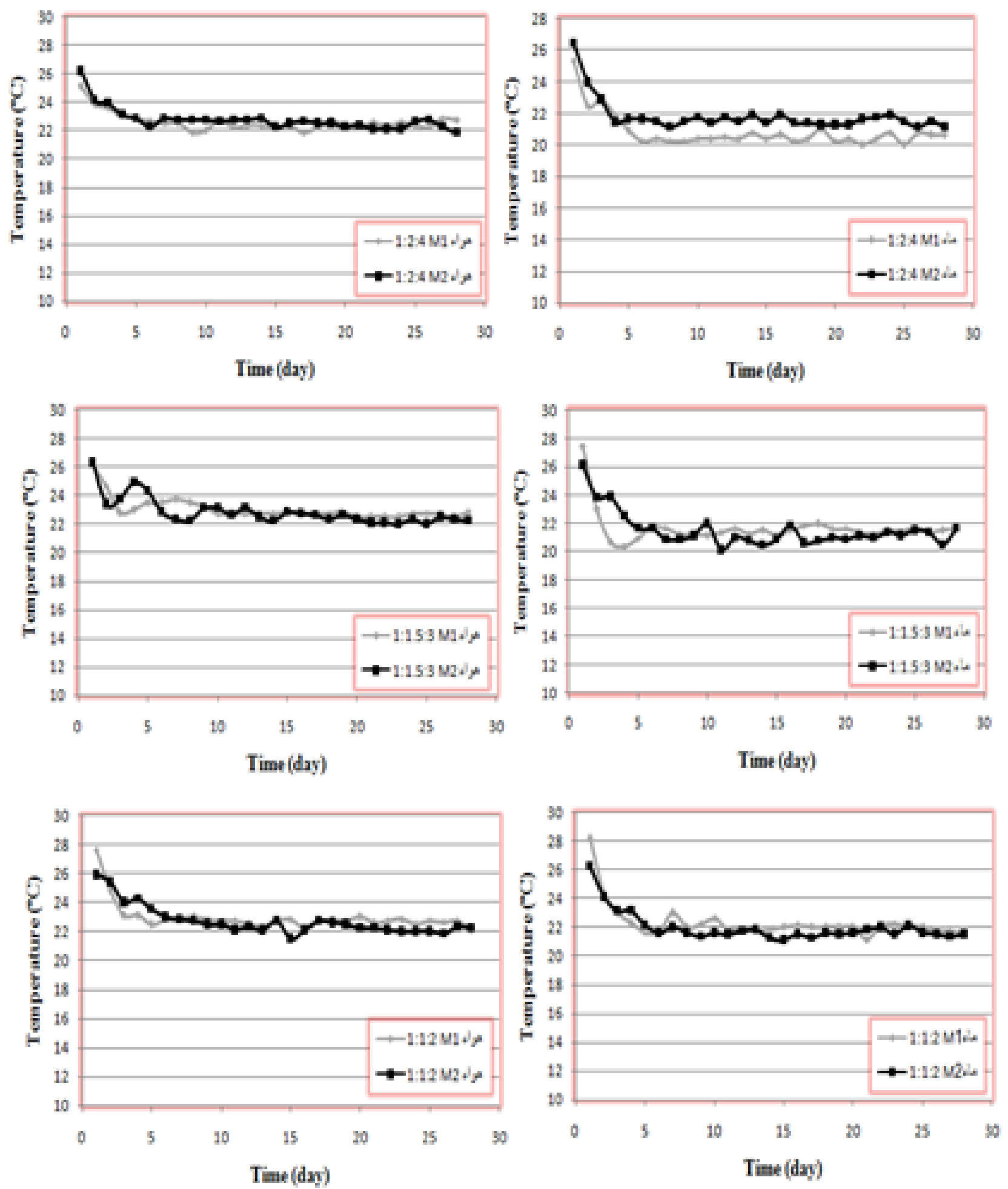

الثكل (6): تأثير وجود السليكا بنسبة (15\%) من وزن الاسنت في حرارة تميؤ الخرسانة لكل نوع من الخلطات 


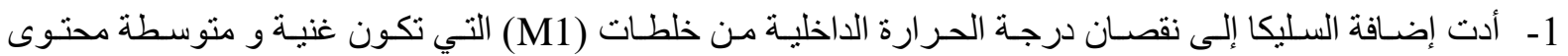

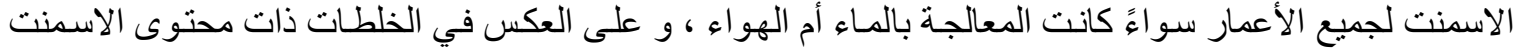

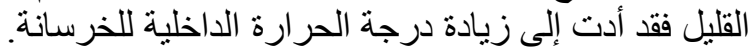

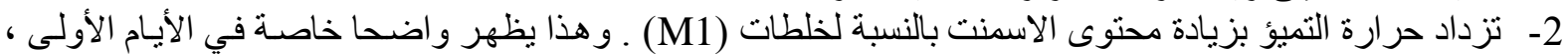

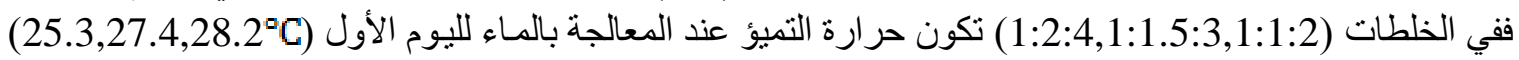

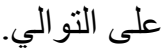

3- إن حر ارة التميؤ لليوم الأول أكثر من الحرارة الناتجة في اليوم الثناني، و هي متذبذبة خـلال (48 سـاعة الأولى) ، ثم التم

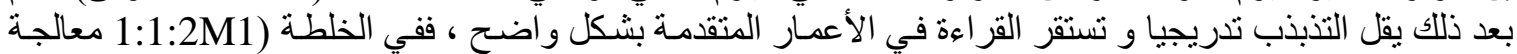

ماء) كان معدل درجة الحر ارة في اليوم الأول (28.2

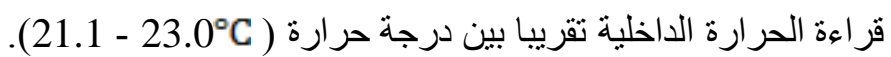

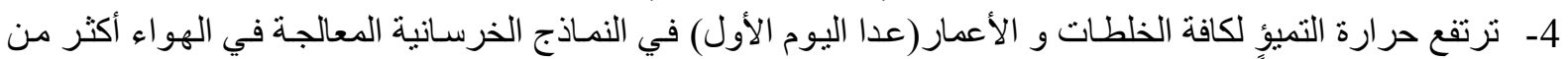

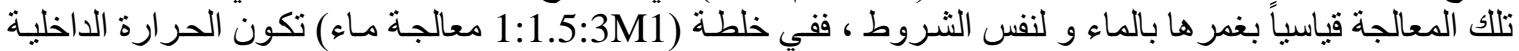
للخرسانة (27.4,23.0º

نفس الخلطة المعالجة في الهواء هي (26.2,24.6º $)$ لليومين (1,2) على التو الي ثم تستقر بين (23.5 (22.5)

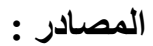

[1] Safiuddin, M., Raman, S. N., and Zain, M. F., "Effect of Different Curing Methods on the Properties of Microsilica Concrete", Australian Journal of Basic and Applied Sciences, No.1(2), 2007, pp. 87-95.

[2] “Concrete Maturity Harnessing The Rate of Strength Change of your concrete", Wisconsin Concrete Pavement Associational, February, 2010.

[3] Bentz, D. P., and Stutzman, P. E., "Curing, Hydration, and Microstructure of Cement Paste", ACI Material Journal, V. 103, No. 5, September-October, 2006, pp.348-356.

المو اصفات القياسية العراقية (رقم 5)، (1984)، "'نصائص الإسدنت البورتلاندي الاعتيادي،،، الجهاز المركزي

للتقييس و السبطرة النوعية، العراق، 1984.

[5] (BS.882-1992), “ Aggregates from Natural Source for Concrete", British Standard Institution, 1992.

[6] ASTM, C 1240-03a , "Standard Specification for Silica Fume Used in Cementitious Mixtures", 2003.

[7] ASTM, C 1074-98, "Standard Practice for Estimating Concrete Strength by the Maturity Method", 1998.

[8] Neville, A. M., Brooks, J. J., "concrete Technology", Longman Scientific \& Technical, Fourth and Final Edition, 2000.

تم اجراء البحث في كلية ألهنسة = جامعة ألموصل 